\title{
63. Experimental Studies on the Generation of Remanent Magnetization of Ferromagnetic Minerals by Chemical Reactions
}

\author{
By Takesi Nagata and Kazuo Kobayashi \\ Geophysical Institute, University of Tokyo \\ (Comm. by S. Nakamura, M.J.A., May 13, 1958)
}

Origin of natural remanent magnetization of rocks has been studied by many investigators. The results show that remanent magnetization of rocks can be classified into three groups, i.e. (a) isothermal remanent magnetization (I.R.M.), being of low magnetic stability, which takes place in any kind of rock containing ferromagnetic minerals; (b) thermo-remanent magnetization (T.R.M.), being of high magnetic stability, which igneous rocks and some metamorphic rocks usually have; and (c) remanent magnetization of sedimentary rocks, which is produced by statistical alignment of ferromagnetic minerals along geomagnetic force during their deposition in lakes, rivers, and the sea. Among these three, (b) and (c) have been used in palaeomagnetism as indication of direction of geomagnetic force at the time of formation of the concerning rocks.

Other possible case of generating remanent magnetization of rocks is the case when chemical reactions or crystallization processes occur in the ferromagnetic minerals contained in rocks under influence of a magnetic field. Although this possibility of remanent magnetization by chemical reactions in nature has already been suggested by Nagata and Watanabe, ${ }^{12}$ Blackett, ${ }^{2)}$ Doell, ${ }^{3)}$ Martinez and Howell, ${ }^{4)}$ and other authors, systematic studies on this problem have only little been done so far. The writers have recently found in laboratory experiments that magnetically stable remanent magnetization can be produced during the reduction process from $\alpha \mathrm{Fe}_{2} \mathrm{O}_{3}$ to $\mathrm{Fe}_{3} \mathrm{O}_{4}$ under influence of magnetic field.

The specimen used in the present study is $\alpha \mathrm{Fe}_{2} \mathrm{O}_{3}$ powder of mean diameter of less than one micron, about two grams being packed in a porcelain boat and is reduced at $340^{\circ} \mathrm{C}$ for twenty hours in the atmosphere of hydrogen gas supplied by Kipp's apparatus under the influence of magnetic field, varying from 0.5 Oe to $40 \mathrm{Oe}$. The temperature $340^{\circ} \mathrm{C}$ was chosen so as the velocity of chemical reaction is sufficiently large in laboratory experiments, and, on the other hand, as low as possible in comparison with the Curie point of $\mathrm{Fe}_{3} \mathrm{O}_{4}$ in order to avoid the influence of the generation of thermo-remanent magnetization. In order to see how the reduction process proceeds, X-ray 
analyses were performed before and after the chemical reaction. It was also confirmed by $\mathrm{X}$-ray analyses that the reduction from $\alpha \mathrm{Fe}_{2} \mathrm{O}_{3}$ to $\mathrm{Fe}_{3} \mathrm{O}_{4}$ is almost completed at $340^{\circ} \mathrm{C}$ in twenty hours under the present experiment conditions.

The remanent magnetization generated in the specimen after the suppression of magnetic field and cooling in field free space was measured by means of an astatic magnetometer at the room temperature, being denoted by $J_{c r, 340^{\circ} \mathrm{C}, H}\left(T_{0}\right)$ and called chemical remanent magnetization. In Fig. 1 the dependence of the chemical remanent magnetization on the intensity of the applied magnetic field is shown. The intensity of the remanent magnetization changes linearly up to

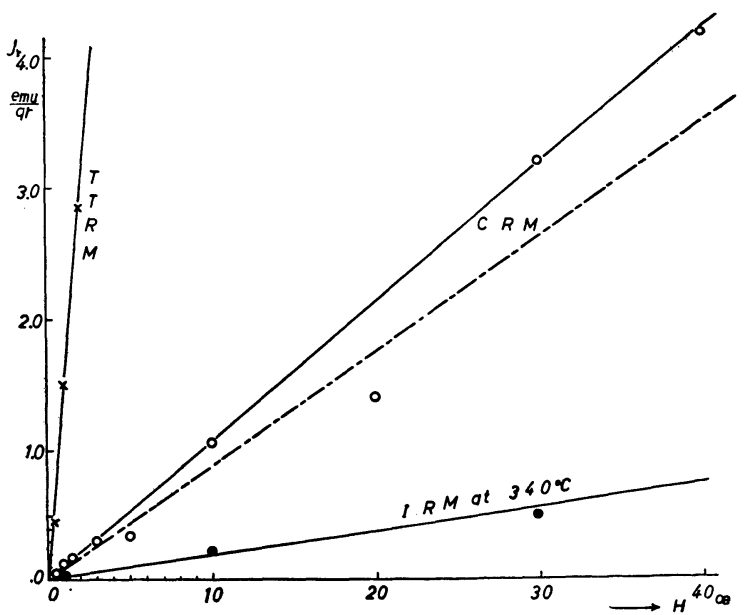

Fig. 1. Intensity of CRM, chemical remanent magnetization, TRM, thermo-remanent magnetization and IRM, isothermal remanent magnetization as dependent on applied magnetic field

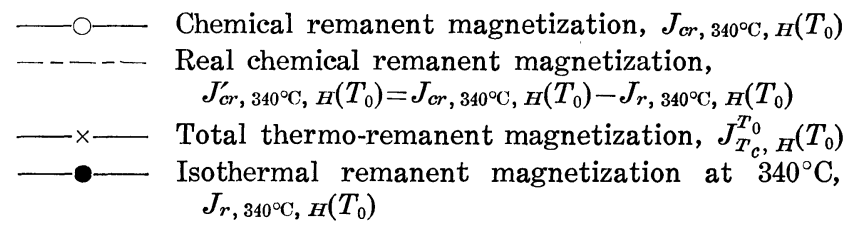

40 Oe according as the field intensity increases. For comparison the total thermo-remanent magnetization $J_{T_{c}}^{T_{0}{ }_{H}}\left(T_{0}\right)$ where $T_{c}$ denotes Curie temperature as well as the isothermal remanent magnetization at $340^{\circ} \mathrm{C} J_{r, 340^{\circ} \mathrm{C}, H}\left(T_{0}\right)$ of the $\mathrm{Fe}_{3} \mathrm{O}_{4}$ powder thus produced is also shown in the figure, where $J_{r, 340^{\circ} \mathrm{C}, H}\left(T_{0}\right)$ indicates the value that was produced in the specimen after the suppression of magnetic field which was acting at $340^{\circ} \mathrm{C}$ for twenty hours, and measured at the room temperature $T_{0}$ after cooling from $340^{\circ} \mathrm{C}$ in field free space. In the said two cases, the $\mathrm{Fe}_{3} \mathrm{O}_{4}$ powder sealed into a silica tube evacuated approximately to $10^{-3} \mathrm{~mm} \mathrm{Hg}$ is used in order to avoid the effect of 
the chemical change through oxidation. The chain line in the figure shows the value of $J_{c r, 340^{\circ}, H_{H}}\left(T_{0}\right)-J_{r, 340^{\circ} \mathrm{C}, H}\left(T_{0}\right)$ which may be considered to be the real chemical remanent magnetization in the exact sense of the word. This will be denoted by $J_{c r}^{\prime}, 340^{\circ} \mathrm{C}, H\left(T_{0}\right)$ and used for the chemical remanent magnetization hereafter in the present paper. It is a remarkable fact that the intensity of $J_{c r, 340^{\circ}, H_{H}^{\prime}}^{\prime}\left(T_{0}\right)$ is several times greater than the isothermal remanent magnetization $J_{r, 340^{\circ} \mathrm{C}, H}\left(T_{0}\right)$ and is about one-tenth of the total thermo-remanent magnetization $J_{T_{c}, H}^{T_{0}}\left(T_{0}\right)$. As for the thermo-remanent magnetization, the effect of the superposition of the isothermal remanent magnetization was ignored, because the intensity of thermo-remanent magnetization is usually a few hundreds times greater than isothermal remanent magnetization.

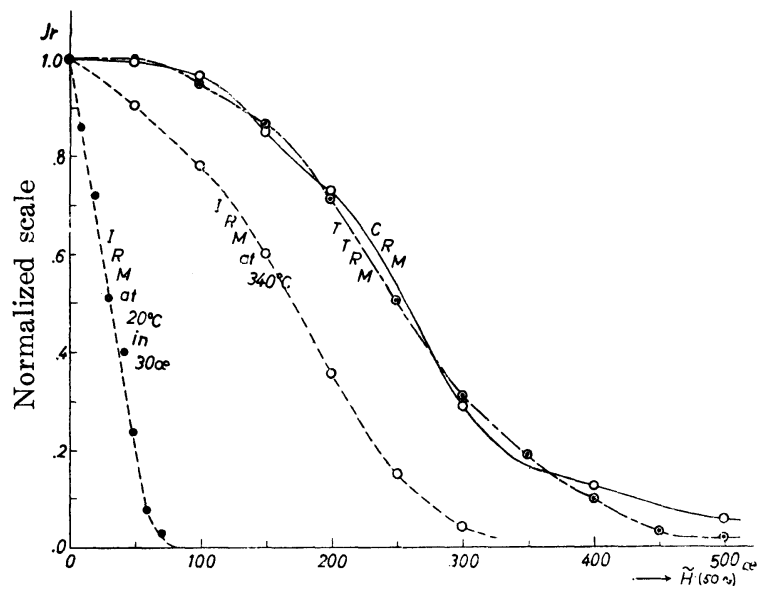

Fig. 2. Different forms of demagnetization curve by alternating magnetic field

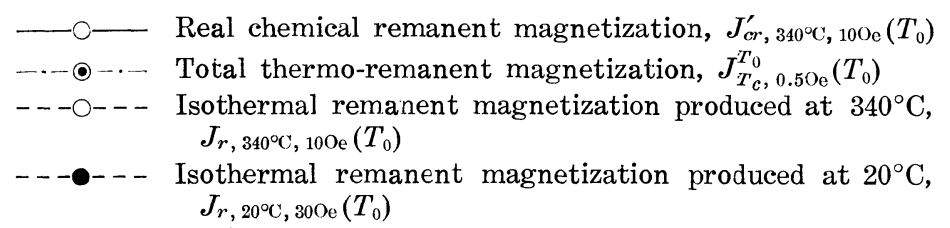

In order to make the distinction of chemical remanent magnetization from the thermo-remanent magnetization and the isothermal remanent magnetization more precisely and to examine stability of the remanent magnetization, demagnetization by alternating magnetic field as well as thermal demagnetization was carried out. The change in the remanent magnetization with the demagnetization process through the alternating magnetic field up to 500 Oe together with the total thermo-remanent magnetization and the isothermal remanent magnetization of the same sample, is shown in Fig. 2, where ordinate indicates the normalized intensity of the remanent magnetization, i.e. $J_{c r, 340 \%, H}^{\prime}(\widetilde{H}) / J_{c r, 340^{\circ} \mathrm{C}, H}^{\prime}(\tilde{H}=0)$ etc. It is clearly seen 
in the figure that behavior of chemical remanent magnetization in the alternating magnetic field is quite similar to that of the thermoremanent magnetization and is much more stable than the isothermal remanent magnetization at the same temperature.

In Fig. 3 the thermal demagnetization curve of the chemical remanent magnetization is shown together with that of the total thermo-remanent magnetization and the isothermal remanent magnetization. It was also confirmed that the chemical remanent magnetization is extremely stable compared with the isothermal remanent magnetization and changes with temperature in almost the same manner as the thermo-remanent magnetization.

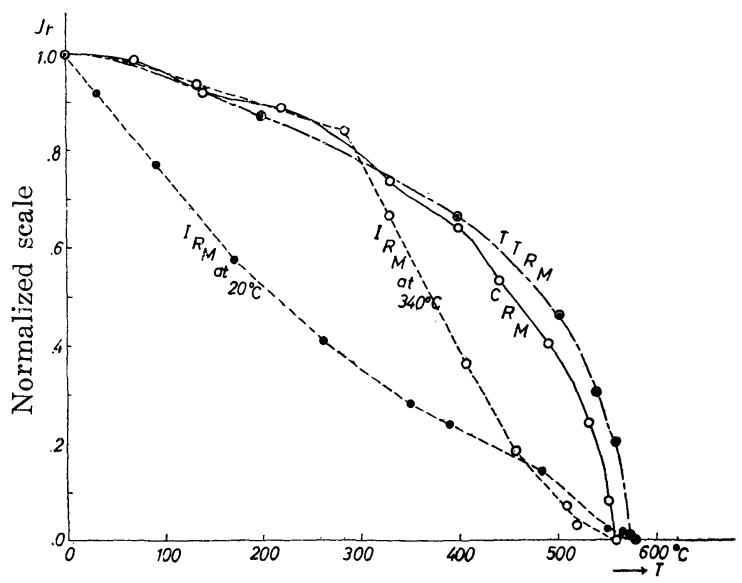

Fig. 3. Thermal variation of remanent magnetizations

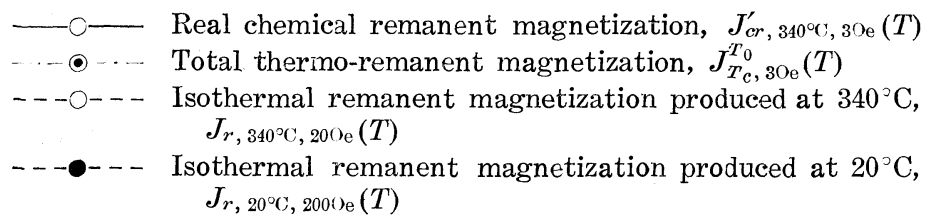

From these experimental results, it may be safely said that the remanent magnetization, of which intensity is intermediate between isothermal remanent magnetization and thermo-remanent magnetization, can be generated by chemical reaction from $\alpha \mathrm{Fe}_{2} \mathrm{O}_{3}$ to $\mathrm{Fe}_{3} \mathrm{O}_{4}$ under the influence of magnetic field. The fact that the magnetic and thermal stability of the chemical remanent magnetization resembles to the thermo-remanent magnetization may suggest that the physical mechanism, through which the chemical remanent magnetization is generated, is nearly the same as that of the thermo-remanent magnetization. In order to get the more general feature on the chemical remanent magnetization, however, further examination must be done under different conditions and with different matters. Some experi- 
ments on the production of remanent magnetization during chemical changes with other transformations such as from $\mathrm{Fe}_{3} \mathrm{O}_{4}$ to $\gamma \mathrm{Fe}_{2} \mathrm{O}_{3}$, from goethite to maghemite, are now undertaken.

\section{References}

1) T. Nagata and T. Watanabe: Geophysical Notes, University of Tokyo, 3, no. 21 (1950); Rock Magnetism, Maruzen, Tokyo (1953).

2) P. M. S. Blackett: Lectures on Rock Magnetism, Weizmann Science Press, Jerusalem (1956).

3) R. Doell: Trans. Amer. Geophys. Union, 37, 156 (1956).

4) J. D. Martinez and L. G. Howell: Nature, 178, 204 (1956). 